\title{
Estructuras musicales en la obra poética de José Ángel Valente: de Schenker a Guillaume de Machaut
}

\section{Musical Structures In the Poetic Work Of José Ángel Valente: From Schenker To Guillaume de Machaut}

\author{
Juan José Pastor Comín \\ Universidad de Castilla-La Mancha \\ Centro de Investigación y Documentación Musical (CIDoM)- \\ Unidad Asociada al CSIC. \\ JuanJose.Pastor@uclm.es \\ ORCID iD: https://orcid.org/0000-0001-8165-1232
}

\section{RESUMEN}

Los textos poéticos no constituyen entidades autorreferenciales limitadas a la propiedades de la palabra como signo lingüístico. Para José Ángel Valente, existe, más allá de la palabra, una estructura musical prelingüistica que contribuye a componer las reglas de interpretación de su obra literaria. Dichas claves musicales son imprescindibles para la comprensión extensa de los posibles sentidos comprendidos en sus versos y su consideración se impone como necesaria. En este trabajo veremos cómo la experiencia musical del poeta se convierte en instrumento decisivo para el ejercicio de la hermenéutica literaria.

Palabras clave: Música y literatura, José Ángel Valente, Schenker, Guillaume de Machaut, Schoenberg, Comparatismo.

\begin{abstract}
Poetic texts are not self-referential entities limited to the properties of the word as a linguistic sign. José Ángel Valente finds, beyond the word, a pre-linguistic musical structure that contributes to composing the rules of interpretation of his literary work. These musical keys are essential for the extensive understanding of the possible meanings included in his verses and their consideration is necessary. In this paper we will see how the poet's musical experience becomes a decisive instrument for the exercise of literary hermeneutics
\end{abstract}

Key words: Music and Literature, José Ángel Valente, Schenker, Guillaume de Machaut, Schoenberg, Comparativism. 


\section{EstruCturas MUSICALES EN LA OBRA POÉTICA DE JosÉ ÁNGEL VALENTE: DE SCHENKER A GUILLAUME DE MACHAUT}

Pastor Comín, J. J. (2018). Estructuras musicales en la obra poética de José Ángel Valente: de Schenker a Guillaume de Machaut. Cuadernos de Investigación Musical, 2018, 6 (extraordinario), 360-372.

doi: 10.18239/invesmusic.v0i0.1952

\section{INTRODUCCIÓN}

En José Ángel Valente (1929-2000) la experiencia estética y, en definitiva, el camino del conocimiento surge de un magma indiferenciado que no se circunscribe a la identidad de cada sistema representacional, sino que más bien se extiende, según veremos más adelante, en la expresión integral de las formas simbólicas. En este sentido la escritura de nuestro poeta, sea cual sea su dimensión en el ámbito público -bien como poemarios (Valente, 2014), bien como ensayos (Valente, 2008) - o privado - a través de la hilazón de un breve diario íntimo (Valente, 2011) - nos ofrece una experiencia singular que lo distancia de sus contemporáneos al filtrar sus vivencias musicales en el espacio de la palabra.

No sorprende, pues, que en sus textos poéticos hallemos una constante contaminación de formas genéricas y estructuras de naturaleza musical que trascienden una mera vacuidad convencional elocutiva y que proyectan sobre el verso y su dimensión formal la tensión vibrante de su ascendente musical primero. Sucede así con poemas como "Salmodia de la buenahombría" o "Sobre el lugar del canto", de Poemas a Lázaro (19551960); "Un canto", de La memoria y los signos (1960-1965); el poemario Breve son (1953-1968), con composiciones como "Canción de espera", "Nana de la mora", "Tres canciones de barcas", "Canción de cuna"; "Tango y perdón", "Himno" o "Fragmento de composición coral", en El inocente (1967-1970); la "Arietta, opus 111" de Interior con figura (1973-1976); el libro Tres Lecciones de Tinieblas (1980) ${ }^{1}$ o Mandorla (1982), este último con su poema "Versión de Trakl-Webern"; o los poemarios No amanece el cantor (1992) y Cantigas de alén (1980-1995), presentados desde sus títulos dentro de un contexto específicamente musical ${ }^{2}$.

Buena parte de los textos de Valente no serían comprendidos si no consideramos su biblioteca personal, especialmente en lo que concierne a la presencia de obras de naturaleza musical, tanto de ediciones, como de estudios teóricos e históricos (Redondo, 2016) ${ }^{3}$. Adorno, Bovet, Barbieri, César Cui, Dufourcq, Druilhe, Fetis, Fubini, Headington, Hindley, Jankelevitch, Martinez Torner, Pedrell, Salazar, Ruiz Tarazona, Satie, Stravinsky o Webern jalonan sus estantes, entre otros nombres. Sin embargo su conocimiento de la Neue Wiener Schule, de la obra de Schoenberg, Berg y, especialmente Webern, así como su estudio nada ingenuo de los fundamentos teóricos de Schenker conceden a su escritura una naturaleza musical todavía no considerada en profundidad ${ }^{4}$.

\footnotetext{
${ }^{1}$ Véase el excelente estudio de Escobar Borrego, 2012: 118-191.

2 Todos ellos recogidos en Valente, 2014.

3 Véase también Redondo, 2016b.

4 Vid. Pastor Comín, 2018b (en prensa). Allí trataremos aspectos aquí apenas esbozados, especialmente en todo aquello que concierne a la presencia de Anton Webern en su pensamiento poético
} 


\section{JUAN JosÉ PASTOR COMÍN}

\section{COUPERIN Y LA URSATZ}

Partamos de una breve anotación en su Diario anónimo que confirma la extraordinaria impronta que en su obra poética dejó un estímulo musical definido: "Enero de 1995. En enero de 1995 interpretaron las Lecciones de Couperin (se apagan 17 cirios) en Versailles" (Valente, 2011: 339). En el año 1980 Valente escribe sus Tres lecciones de tinieblas, a las que regresará en distintas ocasiones en sus textos ensayísticos ${ }^{5}$. Esta obra, extraordinariamente analizada por la crítica ${ }^{6}$, recoge en sus páginas finales - "Tres lecciones de tinieblas: una autolectura"- la clave decodificadora de su propuesta:

Los textos de Tres lecciones de tinieblas tienen su origen en la música. Primero, y antes que en ninguna cosa, en las lecciones de Couperin. Luego, en las de Victoria, Thomas Tallis, Charpentier, Delalande. Del lento depósito de esas composiciones fue desprendiéndose o fomándose un solo principio iniciador o movimiento primario, ese movimiento que subyace en toda progresión armónica y que ha sido llamado justamente Urstaz. En ciertos sistemas de análisis armónicos se entiende que el Ursatz tiene un potencial expresivo universal y, por universal, objetivo. La creación dependería de la medida en que el compositor facilite (por tanteo y por espera) la convergencia de su propia energía con el Ursatz. Lo que en música se llama variación sería, desde ese punto de vista, un modo de meditación creadora sobre el movimiento primario, sobre una forma universal (Valente, 2014: 403).

Valente ha pensado en sucesivas ocasiones sobre la forma poética como trasunto del impulso musical, y autores como Mozart (Valente, 2011: 82-83, 248), Shostakovich (Valente, 2011: 63), Prokofiev (Valente, 2011: 91) o Mahler (Valente, 2011: 111) le sirven para pensar sobre conceptos estructurales tales como la variación o la forma cíclica. Sin embargo, veremos que serán Machaut, Beethoven, Schoenberg y, muy especialmente Webern, quienes propicien un caudal suficiente para configurar su escritura dentro el pensamiento inicial de las formas simbólicas.

La música, como la pintura o el poema se elevan como un objeto mediador entre el hombre y la realidad. Su opacidad y al mismo tiempo revelación formal resuelven el proceso mismo de creación que procede de un sistema de decisiones maduras e improvisadas que avanzan a tientas hacia esa configuración sensible inscrita en la historia del arte, y es, precisamente, esa naturaleza dinámica y conflictiva que subyace a la obra la que interroga y emociona al lector-oyente-espectador:

\footnotetext{
5 "Yo escribí Tres lecciones de tinieblas (Barcelona, La Gaya Ciencia, 1980) a partir de la música de Couperin, pero también ó las voces del mundo musical gregoriano y las de Victoria, Tallis, Charpentier, Delalande. Escribí o escuché las letras, las catorce primeras letras del alfabeto hebreo, de alef a nun. Esos catorce versos son, han sido, huella del rasante paso del viento - ruab- y del espíritu. En ellas está la generación infinita de la materia y de los mundos. No pertenecen al orden de la forma, sino al orden de la formación" ("El maestro de la llama”. Valente, 2008: 703).

${ }^{6}$ Profundícese su estudio en el excelente artículo que contempla esta composición desde una perspectiva musical de Escobar Borrego: 2012: 118-191.
} 


\section{ESTRUCTURAS MUSICALES EN LA OBRa POÉTICA DE JosÉ ÁNGEL VALENTE: DE SCHENKER A GUILLAUME DE MACHAUT}

18 de septiembre de 1960. Es evidente además que la belleza de un cuadro nos conmueve de manera muy distinta a la de belleza natural. Y nos conmueve precisamente por ser el cuadro no un objeto natural sino una relación entre el hombre y la realidad (Valente, 2011: 55).

Por esta razón no es arbitraria, según examinaremos más tarde, su alusión a una Ursatz que guíe la construcción de la forma bajo los principios de la qualitas que él mismo anota de Santo Tomás en su diario: "Las tres cualidades de la obra literaria, según Santo Tomás: Integritas (unidad, totalidad), consonantia (coherencia, decorum), claritas (capacidad de iluminación de la palabra)" (Valente, 2011: 105). Su preocupación en este aspecto es constante y la propia experiencia plástica le conduce a confirmar en la forma la inusitada expresión de cuanto subyace oculto:

9 de noviembre de 1959. "La obra de arte es una construcción de elementos concretos que no adquieren expresividad más que en el proceso de síntesis o de ordenación: la forma de la obra constituye su contenido y toda posible expresividad de la obra de arte no se origina más que con la forma" (Kandisnky en 1912, Sir Herbert Read, A Concise History of Modern Painting, Thames \& Hudson)" (Valente, 2011: 40).

La Ursatz, como directriz armónica es así guía de las resoluciones que el poeta, con oficio de músico, adopta en su personal agonía - à $\gamma \omega v i \alpha$, entendida esta en su sentido griego- por desvelar, tal y como señala en sus Tres lecciones de tinieblas, el "canto de la germinación y del origen, o de la vida como inminencia y proximidad" (Valente, 2011:404). La música adopta así el principio de guía, y el poeta adopta el oficio de espartero -ese tan querido en su Almería adoptada- para acomodar el trenzado de la palabra a una estructura primigenia:

19 de abril de 1987. Cuando en el camino hacia la escritura percibimos un ritmo, una entonación, una nota, algo que es, sin duda, de naturaleza radicalmente musical, algo que remite al número y a la armonía, la escritura ha empezado a formarse. Escribir exige, ante todo, del oído una gran acuidad (Valente, 2011: 246).

Comienza, pues, desde muy pronto a situarse en las polaridades de la repetición y de la variación como fuerzas de construcción en una escritura que tiende cada vez más hacia lo fragmentario, obsesionada por evitar lo iterativo y recurrente. En plena madurez encontramos este desasosiego recogido en su diario:

25 de marzo de 1972. Romper la ley de la repetición como eje formal del lenguaje poético. El principio de la composición lineal se busca en algunos de los poemas recogidos en Treinta y siete fragmentos (Valente, 2011: 151). 


\section{JUAN JOSÉ PASTOR COMÍN}

La variación será, pues, el recurso idóneo para acceder al sentido y el posible título de un libro -finalmente desestimado- para una antología de su poesía en la Editora Nacional, Textos y variaciones (Valente, 2011: 213) ${ }^{7}$. Ahora bien, ¿de dónde tomará Valente los instrumentos adecuados para filtrar su aspiración musical sobre el espacio del verso?

\section{De Guillaume de Machaut a T. S. Eliot: Claves de lectura}

La respuesta la tenemos en Guillaume de Machaut, sobre quien dirige su atención para adoptar sus soluciones formarles y preservar los principios tomistas de la belleza más arriba citados. Confiesa así su fascinación por los movimientos cancrizantes y el contrapunto en espejo ${ }^{8}$, y no duda en hacer en su Diario anónimo una larga anotación que muestra nítidamente la dimensión que este descubrimiento tiene sobre su pensamiento poético:

28 de diciembre de 1959. Guillaume de Machaut (1300-1377). Poeta y músico y el compositor más importante del siglo XIV. Fue durante muchos años secretario de Juan de Luxemburgo, el ciego rey del Bohemia que murió en Crecy.

The History of Music in Sound, Oxford University Press, 1953.

«Ma fin est mon commencement et mon commencement ma fin (My end is my beginning and my beginning my end). This setting of a well-known moralizing motto of the Middle Ages shoes his technical mastery; the music illustrates the words, the cantus (highest part) singing exactly the same notes as the tenor (middle part) but in reverse order, while de contratenor (lowest part) exactly reverses in the B section the order of its own notes in the A. (A and B are really the first sections of the rondeau, the rest of the words giving in enigmatic terms directions for performance).

(The most elaborate form of the song with refrain is the rondeau, in which five poetic verses ( $\mathrm{b} \mathrm{c} \mathrm{d} \mathrm{e)} \mathrm{and} \mathrm{two} \mathrm{strains} \mathrm{of} \mathrm{music} \mathrm{(A} \mathrm{B)} \mathrm{are} \mathrm{fitted} \mathrm{together} \mathrm{in} \mathrm{a} \mathrm{combined}$ pattern:

$$
\begin{aligned}
& \text { Music A B A A B A B } \\
& \text { Words a b c a d e a b » }
\end{aligned}
$$

El motivo del rondeau es el del «East Coker».

Vid. Cap. II en H. Gardner y la opinión de Eliot sobre el tema en «The Music of Poetry» («I think that a poet may gain much from the study of music», etcetera).

El motto en cuestión fue también el lema bordado en el trono de María Estuardo

(Valente, 2011: 45-46).

\footnotetext{
7 Aunque este título no fuera jamás utilizado, si lo empleó para su conferencia en la Fundación General Meditarránea el 29 de mayo de 1981, según recoge el diario $A B C$ de la misma fecha, p. 30.

${ }^{8}$ Esta admiración le acompañará toda su vida: "17 de diciembre de 1994. Si, sólo per speculum in aenigmate" (Valente, 2011: 337, en clara traducción de San Pablo, Corintios I, XIII, 12).
} 


\section{ESTRUCTURAS MUSICALES EN LA OBRA POÉTICA DE JOSÉ ÁNGEL VALENTE: DE SCHENKER A GUILLAUME DE MACHAUT}

La cita es sin duda extensa, pero merece un comentario detenido que nos ilumine tanto sobre sus fuentes como sobre su integración en el contexto de sus otras lecturas. El conocimiento de Machaut le viene por la colección The History of Music in Sound, producida por His Master's Voice (HMV) bajo la dirección de Dom Anselm Hughes y Gerald Abrahm. Si bien este fue un proyecto originario de la BBC Radio, fue editada por HMV en diez volúmenes entre 1953 y 1959. Se publicaron diecinueve horas de música en discos de $78 \mathrm{rpm}$, con libretos explicativos que contenían ejemplos transcritos. Esta edición fue concebida como apoyo en las audiciones de la New Oxford History of Music, cuya andadura se inició apenas un año después, en 1954. El volumen al que se refiere Valente llevaba por título Ars Nova and The Renaissance (c. 1300-1540), tercero de la colección, y recogía de Machaut "Ma fin es mon commencement", con la interpretación instrumental de Clarence Roberts y Lemuel Hughes y un Benedictus cantado por el Brompton Oratory Choir bajo la dirección de Henry Washington". Valente encuentra así el modo de cómo salvar las recurrencias de las formas textuales y musicales en sus singulares combinaciones, clausurando la obra con las correspondencias iniciales. El movimiento palíndromo o retrogradado de las dos voces superiores en "Ma fin est mon commencement / et mon commencement ma fin", así como el regreso sobre sus pasos del tenor una vez que alcanza el punto medio de la canción deforma la concepción del tiempo, haciendo que comienzo y fin convivan tanto sobre la pauta como sobre la experiencia sonora. Origen y final son contenidos en el fluir temporal del mismo modo que vimos sucedía en ese terrible camino desandado en el poema "May Day" tras la pérdida de su hijo Antonio.

Por otro lado, Valente nos trae de nuevo a la memoria los Cuatro cuartetos de T. S. Eliot, evocando el poema "East Coker", segundo de la colección. La alusión, en realidad, no es gratuita: East Coker es una pequeña comunidad al suroeste de Inglaterra a la que pertenecían los antepasados de Eliot y cuya iglesia de Saint Michael and All Angels recibiría años más tarde -en 1965- las cenizas del escritor británico-estadounidense, cerrando así quizá no tan fortuitamente- el círculo de los orígenes ancestrales y el destino final. Lo cierto es que el autor de Las palabras de la tribu encuentra ese vínculo temático entre el rondeau y el pórtico presocrático que Eliot sitúa en el preámbulo a sus poemas, de la voz de Heráclito: "El camino que sube y el que baja son uno y el mismo" (Eliot, 1999: 77) 10. "East Coker" se centra en el poder cíclico del tiempo. El propio nombre del poema -su remoto lugar de origen - revela su inalterado lugar de retorno. "In my beginning is my end" (Eliot, 1999: 98) es el primer verso del poema, un calco, como dice Valente, del lema bordado en el trono de María Estuardo y germen, en definitiva, de un inicio en cuyo seno reside su propio acabamiento. Y cuenta con una excelente factura del siglo XIV de la mano de

\footnotetext{
${ }^{9}$ Los quince discos que contenía este volumen recogía polifonía francesa de los siglos XIII, con las obras "Tant con je vivrai" de Adam de la Halle y "A vous, douce debonaire", de Jehannot de l'Escuerlnot; polifonía francesa del siglo XIV, con las canciones anónimas "Le moulin de Paris" y "Se je chant' main"; el dedicado a Machaut; así como los dedicados a polifonía italiana del siglo XIV, con obras de Giobanni da Cascia, Maestro Piero y Landini; canciones inglesas anónimas (1360-1425); y música litúrgica inglesa del siglo XV, con un disco específico dedicado a Dunstable.

10 Vid. Ellis, 2016: 31-76.
} 


\section{JUAN JOSÉ PASTOR COMÍN}

Machaut. Por esta razón trae a colación el ensayo de Eliot The Music of Poetry en el que alcanza a formular una propuesta que reivindica para su propia escritura:

My purpose here is to insist that a "musical poem" is a poem which has a musical pattern of sound and a musical pattern of the secondary meanings of the words that compose it, and that these two patterns are indissoluble and one (Eliot, 1957: 33).

Valente conoce bien la obra de Eliot y asume en su anotación una de sus ideas fundamentales, aquella que atañe a los principios básicos de la estructura:

There are possibilities for verse which bear some analogy to the development of a theme by different groups of instruments; there are possibilities of transitions in a poem comparable to the different movements of a symphony or a quartet; there are possibilities of contrapuntual arrangement of subject-matter (Eliot, 1957:38).

La musicalidad en el ámbito elocutivo no es algo específicamente relevante contrariamente a la creencia establecida- en el terreno poético, sino la relación y articulación de los contenidos en el ámbito dispositivo. Este planteamiento motriz en el conjunto de toda su obra aparece, efectivamente, recogido por Helen Gardner, tal y como señala en su Diario anónimo, en la primera página del capítulo II "The Music of Four Quartets" de su libro The Art of T. S. Eliot. Allí desarrolla una explicación de cada uno de las cinco partes de cada poema, concebidas como movimientos musicales cuyos temas se presentan bajo el perfil de diferentes desarrollos en cada una de esas composiciones ${ }^{11}$. Justifica este pensamiento que Valente retoma en el libro subrayado sobre sus manos con una larga cita que nuestro poeta brevemente resume en un discreto "la opinión de Eliot sobre el tema":

I think that a poet may gain much from the study of music: how much technical knowledge of musical form is desirable I do not know, for I have not that technical knowledge myself. But I believe that the properties in which music concerns the pet most nearly, are the sense of rhythm and the sense of structure. I think that it might be possible for a poet to work to closely to musical analogies: the result might be an effect of artificiality; but I know that a poem, or a passage of a poem, may tend to realize itself first as a particular rhythm before it reaches expression in words, and that the rhythm may bring to birth the idea and the image; and I do not believe that this is an experience particular to myself. The use of recurrent themes is as natural to poetry as to music (Eliot, 1957: 37).

11 Consúltese Gardner, 1949: 33-56. 


\section{EstruCturas MUSICALES EN LA OBRA POÉTICA DE JOSÉ ÁNGEL VALENTE: DE SCHENKER A GUILLAUME DE MACHAUT}

\section{BEETHOVEN, SCHOENBERG, SCHENKER Y EL SILENCIO}

Esta conciencia musical conduce a Valente, sin duda, en un periodo muy concreto de su vida -entre 1976 y 1977-, a mirar hacia dos titanes que ensancharon las dimensiones formales de la música a través de la transformación del material temático: Beethoven y Schoenberg. Ambos llegaron a una desnudez expresiva que contravenía incluso los recursos instrumentales -es el caso de las últimas sonatas para piano de Beethoven, de una economía inaudita; así como la emancipación sonora y desconexión del lenguaje tonal propiciada ya en la Gran fuga Op. 133. Frente a Valente se erige la exigencia de "plantear de otra manera el problema de forma y contenido. Palabra falta no tanto de contenido como de interioridad" (Valente, 2011: 174) ${ }^{12}$. Será, en consecuencia, en el desarrollo de la variación que Beethoven llevará al extremo en sus Variaciones Diabelli, op. 120- donde, como dice el poeta en su ensayo "Modernidad y Postmodernidad: el ángel de la historia", el compositor alemán, junto al Tao te King, la Odisea, la Eneida o San Juan de la Cruz, forma parte de la textura de hilos y vías que conducen a la "querencia" en virtud de la "infinita progresión de la materia y de las formas" (Valente, 2008: 1357) presente y manifiesta en el conjunto de su obra y, muy especialmente, en su sonata para piano op. 111. De todos es sabido que esta Sonata en do menor n. 32 , última escrita para el piano (concebida junto a las op. 109 y 110), tuvo sus primeros esbozos, coetáneos a su Segunda Sinfonía, en 1801-1802. El mismo Schenker, en su estudio Die letzen Sonaten von Beethoven: Kritische Ausgabe mit Einführung und Erläuterung dedicado al examen de las sonatas op. 109, 110, 111 y 101, enterrará la semilla de su concepto Urstaz en el análisis que hace de estas sonatas, tecnicismo más arriba utilizado por Valente y del que hablaremos más tarde ${ }^{13}$. Los conceptos de acorde gramatical y acorde significante, así como la distinción entre acordes de estructura y acordes de prolongación propuestos por el teórico discípulo de Bruckner, han constituido como se sabe, la piedra angular en el último cuarto de siglo Xx del análisis de la música tonal. El pensamiento schenkeriano alcanza en Valente la dimensión de energía emotiva, esto es, inscrita en un marco universal donde el principio de variación - esto es, la no repetición sino, antes bien, la renovación de lo dado- converge sobre dos aspectos esenciales: el primero, su concepto de lectura e interpretación creadora, altamente intelectualizado; en segundo lugar, sobre un mismo objeto artístico, paradigma de la variación en la expresión definitiva del estilo tardío beethoveniano -desnudo y sin concesiones retóricas- su Sonata para piano op. 111.

de julio de 1974. La Ursatz «movimiento primario» o «principio iniciadon», subyace en toda progresión armónica (ver The Beethoven Companion, p. 149, nota). Sistema de análisis armónico de Schenker. El Urstatz tiene un potencial expresivo universal (y, por universal, objetivo). La penetración depende de la medida en que el compositor pueda investir el Ursatź de su propia energía emotiva. La variación universal sería una forma de meditación creadora sobre una forma universal. Es la penetración absoluta en el movimiento primario la que genera esa doble apertura -en profundidad y hacia lo alto- de la Sonata op. 111 (Valente, 2011: 159).

\footnotetext{
12 Anotación del 21 de mayo de 1977.

${ }^{13}$ Consúltese Schenker, 2015: 9-ss.
} 


\section{JUAN JOSÉ PASTOR COMÍN}

Aparece, pues, un nuevo matiz en la construcción formal del significante poético, dado que se inviste de un valor antropológico universal que conlleva el ingreso de la identidad -la experiencia personal- en el acervo expresivo colectivo, de modo que desde un discreto paso anónimo - una de las preocupaciones de Valente en toda su escritura, atributo que dio nombre a sus papeles "privados"- germina y procura la transformación de lo común y propio, del tema dado y conocido. Este es el sentido de disolución conforme a la síntesis del Ursatz shenkerinano:

22 de septiembre de 1974. Disolver en el contenido de la tradición la experiencia personal, con lo que la tradición no se repite, sino que se continúa o se recomienza.

Disuelta en el contenido de la tradición, la experiencia personal es anónima (lo es de todos los hombres).

Ese punto de disolución sería el punto de incidencia de la visión particular con el potencial expresivo universal (con el movimiento primario, con el Urstat:)

(Valente, 2011: 160).

Unos años después veremos cómo esta fascinación por las herramientas intelectuales propiciadas por Schenker y que subyacen a una concepción extensa y al mismo tiempo rigurosa del principio de variación reaparece en nuestra poeta, esta vez sin duda referida nuevamente a su preocupación por pensar la materia propia de la música, impresionado por la naturaleza del op. 111, motor que, según vemos, ha desencadenado desde largo este pensamiento complejo:

20 de mayo de 1977. Sonata 111. Reflexión sobre un intervalo. Aparece al abrir. Se invierte en el tiempo lento. Reaparece al final. Reflexión sobre la materia de la música, que es reflexión sobre la materia total del mundo (Valente, 2011: 174) ${ }^{14}$.

Esta anotación es contemporánea a su libro Interior con figuras (1976), donde integra en su Parte IV el poema Arietta, op. 111. Si bien a nuestro juicio la crítica ha errado en la ubicación del referente sonoro -aplicado equívocamente a toda la sonata, y no, como concierne, solo al segundo movimiento ${ }^{15}-$, lo cierto es que, cuando menos, se ha reconocido en este poema un ejercicio de escritura encaminado a liberar la forma desde la experiencia musical vivida. Dividido el poema en cinco secciones, recogemos aquí las dos primeras y la última:

\footnotetext{
${ }^{14}$ Esta reflexión de Valente nos recuerda sin duda a la que Thomas Mann incluye sobre esta misma sonata en el Capítulo VIII de su novela Doktor Faustus. Das Leben des deutschen Tonsetzers Adrian LeverKühn, erzälht von einem Freunde (1947).

${ }_{15}$ Consúltese Gallego, 1996: 32-33, donde discute algunos trabajos anteriores que toman como referencia del poema distintas partes de la Sonata op. 111.
} 


\section{EstruCturas MUSICALES EN LA OBRA POÉTICA DE JOSÉ ÁNGEL VALENTE: DE SCHENKER A \\ GUILLAUME DE MACHAUT}

1

Forma

(en lo infinitamente abierto hacia lo informe).

2

El silencio se quiebra

en trino por tres veces

y la materia de la música ya no es sonido sino transparencia.

$[\ldots]$

5

Para que el hilo tenue tan infinitamente se prolongue,

para que solo quede por decir

la total extensión de lo indecible,

para que la libertad se manifieste,

para que andar del otro lado de la muerte sea

semplice e cantábile

y aquí y allí la música nos lleve

al centro, al fuego, al aire,

al agua antenatal que envuelve

la forma indescifrable

de lo que nunca nadie aún ha hecho

nacer en la mañana del mundo

(Valente, 2014: 353-364).

El poema de Valente, al igual que la Arietta (cinco variaciones sin solución de continuidad), emerge libre de todo vínculo formal y comparte con él un carácter errático. La condición armónica casi primitiva de la música beethoveniana se resuelve aquí en un verso desnudo, despojado de cualquier embellecimiento elocutivo superficial. Del mismo modo que en la pieza para piano la melodía se disuelve a través del ritmo y de los aglomerados de acordes que la sostienen, el poema nos regresa aquí al "agua prenatal" y busca el "nacer en la mañana del mundo" de un modo similar a la manera en que el tema de la arietta emerge del trino constante de su quinta variación y, muy especialmente, en sus compases finales.

La segunda gran figura a la que Valente dirige su mirada es Arnold Schoenberg, autor de las Variaciones para orquesta op. 31 -referencia ineludible en el estudio de la forma variación-, estrenadas en 1928 por Wilhelm Furwängler. De su interés por el compositor que buscó la renovación del lenguaje musical dentro del compromiso con el temperamento igual han quedado unas anotaciones en su Diario anónimo días después de sus reflexiones sobre Beethoven: 


\section{JUAN JosÉ PASTOR COMÍN} Boulez).

10 de junio de 1977. Schönberg, Gurre-Lieder (2 discos, CBS, dirección de Pierre

Los "Cantos de Gurre", traducidos del danés de Jens Peter Jacobsen (el amor del rey Waldemar por Tove, la reina celosa mata a Tove; la blasfemia del rey, condenado a cazar eternamente en los bosques nocturnos), Schönberg emplea aquí por vez primera el Sprechgesang: "Cazar salvaje del viento de estío" (los fantasmas nocturnos se disipan y se anuncia la apoteosis del sol).

Moisésy Arón se estrenó en Hamburgo en 1954. (Schönberg, 1874-1951).

Inicio: el diálogo en que se oponen Moisés y Arón (la irreductibilidad del pensamiento puro, la visión absoluta de lo divino incomunicable; la técnica del poder; la demagogia de la comunicación, la fijación de imágenes, el Becerro de Oro)".

Moises: $O$ Wort, du Wort, das mir febtl! (jOh palabra, palabra, tú que me abandonas!)

(La ópera está inacabada, falta el tercer acto)

(Valente, 2011: 175-176).

La grabación que encabeza esta entrada y motivó la reflexión del poeta fue realizada por la BBC Symphony Orchestra bajo la dirección de Pierre Boulez ${ }^{16}$. Sorprende que sean los Gurrelieder-oratorio monunmental para cinco voces, narrador, coros y orquesta- el origen de un hilo ha de conducir, apenas un año más tarde, hacia una breve nota en la que Webern toma el testigo del silencio y las formas concisas:

11 de marzo de 1978. Schönberg, Tres piezas breves, 1910.

Concreción extrema. Economía de medios. No repetición.

El sentido en que opera Webern. Es el mismo momento en que se sitúa el comienzo de la tercera y más radical fase de Webern: Cinco piezas para cuarteto de cuerda (1909), Seis piezas para orquesta (1910), Cuatro piezas para violin y piano (1910).

La «forma menor»: concentración y transparencia. (La presencia del haiku). Vía hacia la composición del silencio: Trío de cuerda (1927), Concierto para nueve instrumentos (1934).

Coloración instrumental, amplios intervalos y pasajes enteramente silenciosos

(Valente, 2011: 177).

\footnotetext{
${ }^{16}$ Editado en dos vinilos por Columbia Masterworks (M2 33303), Pierre Boluez Conducts Schoenberg - Gurre-Lieder fue publicado en 1975 y registrado por la BBC Symphony Orchestra y una amplia intervención en las partes corales de la BBC Choral Society, la BBC Singers, los Gentlement of the London Pbilarmonic Choir y las Goldsmith's Choral Union bajo la dirección de John Pool. Los solistas fueron la soprano Marita Napier, los tenores Kenneth Bowen, Günter Reich y Jess Thomas, y el barítono Siegmund Nimsgern.
} 


\section{EstruCturas MUSICALES EN LA OBRA POÉTICA DE JOSÉ ÁNGEL VALENTE: DE SCHENKER A GUILLAUME DE MACHAUT}

Parece, sin duda, una evolución lógica cuyo germen fue enterrado en la palabra incapacitada para expresar lo trascendente, dramáticamente expresada en el Moisés y Aarón de Schoenberg ${ }^{17}$. Se extiende así un velo sobre el conjunto de su obra poética que, desde la música -arte que se construye sobre el silencio, hecha de silencios, y extinguida en el silencio- aspira a regresar a esa matriz de ausencias que es a su vez tierra firme y horizonte: "La palabra significa al silencio del que nace y al que siempre remite" (Valente, 2011: 297) ${ }^{18}$.

\section{BIBLIOGRAFÍA}

Eliot, T. S. (1957). On Poetry and Poets. London: Faber and Faber.

Eliot, T. S. (1999). Cuatro cuartetos. Madrid: Cátedra, Letras Universales.

Ellis, S. (2016). The English Eliot: Design, Language and Landscape in "Four Quartets". London \&

New York: Routledge Library Editions.

Escobar Borrego, F. J. (2012). "Tres lecciones de tinieblas, de José Ángel Valente: naturaleza musical, claves de poética e implicaciones simbólicas”, Entymema VI, pp. 118-191.

Gallego, A. (1996). Noche Serena: Glosas contemporáneas a Fray Luis. Discurso del académico electo. Madrid: Real Academia de Bellas Artes de San Fernando.

Gardner, H. (1949). The Art of T. S. Eliot. London: The Cresset Press.

Pastor Comín, J. J. (2018). "El juego del poeta: la música en el verso”. En Segarra, M. D. (ed.). El juego de los sonidos. Homenaje a Antonio Alcázar. Madrid: Alpuerto (en prensa).

Pastor Comín, J. J. (2018). Música y poesía en José Ángel Valente. Vigo: Academia del Hispanismo (en prensa)

Redondo, F. X. (ed) (2016a). Biblioteca de José Ángel Valente. Santiago de Compostela: Universidade de Santiago de Compostela.

Redondo, F. X. (ed) (2016b). Biblioteca de José Ángel Valente [CD]. Santiago de Compostela: Universidade de Santiago de Compostela.

\footnotetext{
${ }^{17}$ En "El maestro de la llama" de La experiencia abisal (1978-1999) Valente se detiene nuevamente sobre el Moisés y Aarón de Schoenberg: "La ópera de Schönberg está inacabada; falta el tercer acto: $O$ Wort, du Wort, das mir felt! Falta la palabra. La visión absoluta de lo divino es incomunicable, el pensamiento es irreductible a las técnicas del poder, a la demagogia de la comunicación” (Valente, 2008: 702).

18 Anotación del 19 de octubre de 1991.
} 


\section{JUAN JOSÉ PASTOR COMÍN}

Schenker, H. (2015). Piano Sonata in C Minor, Op. 111: Beethoven's Last Piano Sonatas, An Edition with Elucidation, Traducción de John Rothgeb. Oxford: Oxford University Press.

Valente, J. Á. [et al.] (1995). IX Xornadas de Música Contemporánea: Anton Webern (1883-1945): universo musical e influencias no $50^{\circ}$ aniversario da sáu norte. Santiago de Compostela: Centro Gallego de Arte Contemporánea.

Valente, J. Á. (2008). Ensayos. Obras Completas II. Barcelona: Galaxia Gutenberg.

Valente, J. Á. (2011). Diario anónimo (1959-2000). Barcelona: Galaxia Gutenberg.

Valente, J. Á. (2014). Poesía completa. Barcelona: Galaxia Gutenberg.

Webern, A. (1924). 6 Lieder nach Gedichten von Georg Trakl, Op.14. Vienna: Universal Edition.

Fecha de recepción: 01/06/2018

Fecha de aceptación: 10/09/2018 